\title{
A New Generalized Algebraic Method and its Application in Nonlinear Evolution Equations with Variable Coefficients
}

\author{
Cheng-Lin Bai ${ }^{\mathrm{a}}$, Cheng-Jie Bai ${ }^{\mathrm{b}}$, and Hong Zhao ${ }^{\mathrm{a}}$ \\ a Physics Science and Information Engineering School, Liaocheng University, \\ Liaocheng 252059, China \\ b Communication School, Shandong Normal University, Jinan, 250014, China \\ Reprint requests to Dr. C.-L.B.; E-mail: 1cced_bcl@1ctu.edu.cn or lcced_bcl@hotmail.com
}

Z. Naturforsch. 60a, 211 - 220 (2005); received December 22, 2004

In this paper, a new generalized algebraic method is proposed to construct a series of explicit exact solutions of general nonlinear evolution equations with variable coefficients. Compared with most existing methods, such as the tanh method, the extended tanh method, the Jacobi elliptic function method or the algebraic method, the proposed method seems to be more powerful. The efficiency of the method is demonstrated by applying it to the (1+1)-dimensional variable coefficients modified Korteweg-de Vries (MKdV) equation and the (2+1)-dimensional variable coefficients KadomtsevPetviashvili (KP) equation. A rich variety of new exact explicit solutions has been found. - PACS numbers: 03.40.Kf; 03.65.Fd; 02.30Jr.

Key words: New Generalized Algebraic Method; Variable Coefficients Nonlinear Evolution Equations; Exact Explicit Solutions.

\section{Introduction}

Many phenomena in physics and other fields are described by nonlinear partial differential equations (PDEs). When we want to understand the physical mechanism of phenomena in nature, described by nonlinear PDEs, exact solutions for the nonlinear PDEs have to be explored. Thus the methods for deriving exact solutions for the governing equations have to be developed. To study exact solutions of nonlinear PDEs has become one of the most important topics in mathematical physics. In the past decades, various powerful methods have been proposed, namely the inverse scattering method, Hirota's bilinear method, the Bäcklund transformation method, the Darboux transformation method, the tanh method, the variable separation approach and the homogeneous balance method. Among those, the tanh method [1] provides a particularly straightforward and effective algorithm to obtain solutions for a large number of nonlinear PDEs. Based on the fact that soliton solutions are essentially of a localized nature, one can write the solitary wave solution of a nonlinear equation as a polynomial of hyperbolic functions and transform it into a nonlinear system of algebraic equations. In recent years, much research work has been concentrated on the various extensions and applications of the tanh method [2-4]. The basic purpose of these papers is to simplify the routine calculation of the method and to find more general exact solutions.

In this paper, we shall develop a new generalized algebraic method using symbolic computation, which greatly exceeds the applicability of the existing tanh, extended tanh, Jacobi function expansion, and some other sophisticated methods in obtaining a series of exact solutions of nonlinear equations. We can use the method to reduce a nonlinear equation to a system of algebraic equations by applying an ordinary differential equation. The characteristic feature of our method is that, without much extra effort, we circumvent the integration by directly getting a series of exact solutions using an uniform way, which readily covers all results of the tanh, the extended tanh, the Jacobi function expansion and some other sophisticated methods. Another advantage of our method is that it is independent of the integrability of the given nonlinear equations. Viewed as a special case of PDEs, the method also applies to nonlinear ordinary differential equations (ODEs).

The paper is organized as follows. In Section 2, the key idea of the new generalized algebraic method is described. In Section 3 and Section 4, the pro- 
posed method is applied to the $(1+1)$-dimensional variable coefficients modified Korteweg-de Vries (MKdV) equations and to the $(2+1)$-dimensional variable coefficients Kadomtsev-Petviashvili (KP) equations. We conclude the paper in the last section.

\section{Summary of the Generalized Algebraic Method}

Here we first describe the key idea of our method. For a given system of nonlinear evolution equations (NEEs) with independent variables $x=\left(x_{0} \equiv t, x_{1}\right.$, $\left.x_{2}, \ldots, x_{n}\right)$ and dependent variables $u, v$

$$
\begin{aligned}
& P\left(x_{0} \equiv t, x_{1}, x_{2}, \ldots, x_{n}, u, v, u_{x_{i}}, v_{x_{i}},\right. \\
& \left.\quad u_{x_{i} x_{j}}, v_{x_{i} x_{j}}, \ldots, u_{x_{i_{1}} x_{i_{2}} \ldots x_{i_{n}}}, u_{x_{i_{1}} x_{i_{2}} \ldots x_{i_{N}}}\right)=0, \\
& Q\left(x_{0} \equiv t, x_{1}, x_{2}, \ldots, x_{n}, u, v, u_{x_{i}}, v_{x_{i}},\right. \\
& \left.\quad u_{x_{i} x_{j}}, v_{x_{i} x_{j}}, \ldots, u_{x_{i_{1}} x_{i_{2}} \ldots x_{i_{n}}}, u_{x_{i_{1}} x_{i_{2}} \ldots x_{i_{N}}}\right)=0 .
\end{aligned}
$$

In general, the left-hand side of (1a) and (1b) is a polynomial in $u, v$ and its various derivatives. Our method for solving (1) proceeds in the following three steps:

Step 1. We assume that (1a) and (1b) have solutions of the form

$$
\begin{aligned}
& u=A_{0}(x)+\sum_{i=1}^{M}\left(A_{i}(x) P^{i}+B_{i}(x) P^{-i}\right), \\
& v=a_{0}(x)+\sum_{j=1}^{N}\left(a_{j}(x) P^{j}+b_{j}(x) P^{-j}\right),
\end{aligned}
$$

where $M$ and $N$ are integers to be determined by balancing the highest order nonlinear terms and the highest order partial derivative terms in (1), $P=P(\xi)$ and $\xi=\xi(x)$ are all differentiable functions, and $P(\xi)$ is a solution of the first-order nonlinear ordinary differential equation

$$
P^{\prime}(\xi)=\varepsilon \sqrt{\sum_{i=0}^{n} c_{i} P^{i}(\xi)}
$$

where $\varepsilon= \pm 1$. The positive integer $n$ and the constants $c_{i}(i=0,1, \ldots n)$ are to be determined. Then the derivatives with respect to the variable $\xi$ become derivatives with respect to the variable $P$ as:

$$
\frac{\mathrm{d}}{\mathrm{d} \xi} \rightarrow \varepsilon \sqrt{\sum_{i=0}^{n} c_{i} P^{i}} \frac{\mathrm{d}}{\mathrm{d} P}
$$

$$
\frac{\mathrm{d}^{2}}{\mathrm{~d} \xi^{2}} \rightarrow \varepsilon^{2}\left[\frac{1}{2} \sum_{i=1}^{n} i c_{i} P^{i-1} \frac{\mathrm{d}}{\mathrm{d} P}+\sum_{i=0}^{n} c_{i} P^{i} \frac{\mathrm{d}^{2}}{\mathrm{~d} P^{2}}\right], \ldots
$$

Step 2. Substituting (2a) and (2b) into the given NEEs (1) and making use of (3), collecting all the terms with the same order of $P^{k}$ and $P^{k} \sqrt{\sum_{i=0}^{n} c_{i} P^{i}}$ (where $i=0,1, \ldots n$ ), and setting the coefficients of each order of $P^{k}$ and $P^{k} \sqrt{\sum_{i=0}^{n} c_{i} P^{i}}$ to zero, yields a set of over-determined partial differential equations with respect to the differential functions $A_{i}(x), B_{i}(x)$, $a_{j}(x), b_{j}(x)$ (where $\left.i=0,1,2, \ldots M ; j=0,1,2, \ldots N\right)$ and $\xi(x)$.

Step 3. Solving the over-determined partial differential equations obtained in step 2 by using symbolic computation packages like Maple or Mathematica, will lead to explicit expressions for $A_{i}(x), B_{i}(x)$, $a_{j}(x), b_{j}(x)$ (where $\left.i=0,1,2, \ldots M ; j=0,1,2, \ldots N\right)$, and $\xi(x)$ or the constraints among them. We remark here that the exact solutions of (1) depend on the explicit solvability of (3). The solution of the system of algebraic equations will become tedious with the increase of the values of $M, N$, and $n$. If $n=4$, (3) gives a series of fundamental solutions such as polynomial, exponential, soliton, rational, triangular periodic, Jacobi and Weierstrass doubly periodic solutions. We consider only the case $n=4$ in this paper and take

$$
\begin{aligned}
P^{\prime}(\xi)=\varepsilon\left\{c_{0}\right. & +c_{1} P(\xi)+c_{2} P^{2}(\xi) \\
& \left.+c_{3} P^{3}(\xi)+c_{4} P^{4}(\xi)\right\}^{1 / 2}
\end{aligned}
$$

By considering the different values of $c_{0}, c_{1}, c_{2}, c_{3}$, and $c_{4}$, we find that (4) admits a series of fundamental solutions, which are displayed in Table 1.

Remark 1. The algorithm is more powerful than the tanh method [1], the extended tanh method [2], the Jacobi function expansion method [3], and the algebraic method [4]. To demonstrate this, we argue here that various previously introduced methods are included in our more general intermediate equation (4). Let us consider the special cases $A_{i}(x)=A_{i}, a_{j}(x)=a_{j}$, $B_{i}(x)=b_{j}(x)=0$ (where $A_{i}$ and $a_{j}$ are constants and $i=0,1,2, \ldots M ; j=0,1,2, \ldots N)$ and $\xi(x)=k_{0} t+$ $k_{1} x_{1}+k_{2} x_{2}+\ldots+k_{n} x_{n}+\varphi$ (where $k_{0}, k_{1}, k_{2}, \ldots, k_{n}$, and $\varphi$ are constants). Then our approach reduces to the algebraic method proposed by Fan [4]. Also, for the case $c_{1}=c_{3}=0, c_{0}=c_{4}=1$, and $c_{2}=2$, (4) has the solution $\tanh \xi$ and our method reduces to the tanh method [1]. In the case when $c_{1}=c_{3}=0, c_{0}=c_{2}^{2} / 4$, and $c_{4}=1$, (4) degenerates to a Riccati equation. In 


\begin{tabular}{|c|c|c|c|c|c|}
\hline$c_{0}$ & $c_{1}$ & $c_{2}$ & $c_{3}$ & $c_{4}$ & $P$ \\
\hline 0 & 0 & $>0$ & 0 & $<0$ & $\varepsilon \sqrt{-\frac{c_{2}}{c_{4}}} \operatorname{sech}\left(\sqrt{c_{2}} \xi\right)$ \\
\hline$\frac{c_{2}^{2}}{4 c_{4}}$ & 0 & $<0$ & 0 & $>0$ & $\varepsilon \sqrt{-\frac{c_{2}}{2 c_{4}}} \tanh \left(\sqrt{-\frac{c_{2}}{2}} \xi\right)$ \\
\hline 0 & 0 & $<0$ & 0 & $>0$ & $\varepsilon \sqrt{-\frac{c_{2}}{c_{4}} \sec \left(\sqrt{-c_{2}} \xi\right)}$ \\
\hline$\frac{c_{2}^{2}}{4 c_{4}}$ & 0 & $>0$ & 0 & $>0$ & $\varepsilon \sqrt{\frac{c_{2}}{2 c_{4}}} \tan \left(\sqrt{\frac{c_{2}}{2}} \xi\right)$ \\
\hline 0 & 0 & 0 & 0 & $>0$ & $-\frac{\varepsilon}{\sqrt{c_{4}} \xi}$ \\
\hline$-\frac{c_{2}^{2} m^{2}\left(1-m^{2}\right)}{c_{4}\left(2 m^{2}-1\right)^{2}}$ & 0 & $>0$ & 0 & $<0$ & $\varepsilon \sqrt{-\frac{c_{2} m^{2}}{c_{4}\left(2 m^{2}-1\right)}} \operatorname{cn}\left(\sqrt{\frac{c_{2}}{2 m^{2}-1}} \xi\right.$ \\
\hline$\frac{c_{2}^{2}\left(1-m^{2}\right)}{c_{4}\left(2-m^{2}\right)^{2}}$ & 0 & $>0$ & 0 & $<0$ & $\varepsilon \sqrt{-\frac{c_{2}}{c_{4}\left(2-m^{2}\right)}} \mathrm{dn}\left(\sqrt{\frac{c_{2}}{2-m^{2}}} \xi\right)$ \\
\hline$\frac{c_{2}^{2} m^{2}}{c_{4}\left(m^{2}+1\right)^{2}}$ & 0 & $<0$ & 0 & $>0$ & $\varepsilon \sqrt{-\frac{c_{2} m^{2}}{c_{4}\left(m^{2}+1\right)}} \operatorname{sn}\left(\sqrt{-\frac{c_{2}}{m^{2}+1}} \xi\right)$ \\
\hline 0 & 0 & $>0$ & $\neq 0$ & 0 & $-\frac{c_{2}}{c_{3}} \operatorname{sech}^{2}\left(\frac{\sqrt{c_{2}}}{2} \xi\right)$ \\
\hline 0 & 0 & $<0$ & $\neq 0$ & 0 & $-\frac{c_{2}}{c_{3}} \sec ^{2}\left(\frac{\sqrt{ }-c_{2}}{2} \xi\right)$ \\
\hline 0 & 0 & 0 & $\neq 0$ & 0 & $\frac{1}{c_{3} \xi^{2}}$ \\
\hline$\neq 0$ & $\neq 0$ & 0 & $>0$ & 0 & $\begin{array}{c}\wp\left(\frac{\sqrt{ } c_{3}}{2} \xi, g_{2}, g_{3}\right) \\
c_{2} \sec ^{2}\left(\frac{1}{2} \sqrt{-c_{2}} \xi\right)\end{array}$ \\
\hline 0 & 0 & $<0$ & & & $\begin{array}{c}2 \varepsilon \sqrt{-c_{2} c_{4}} \tan \left(\frac{1}{2} \sqrt{-c_{2}} \xi\right)+c_{3} \\
c_{2} \operatorname{sech}^{2}\left(\frac{1}{2} \sqrt{c_{2}} \xi\right)\end{array}$ \\
\hline 0 & 0 & $>0$ & & & $2 \varepsilon \sqrt{c_{2} c_{4}} \tanh \left(\frac{1}{2} \sqrt{c_{2}} \xi\right)-c_{3}$ \\
\hline$>0$ & 0 & 0 & 0 & 0 & $\varepsilon \sqrt{c_{0}} \xi$ \\
\hline & $\neq 0$ & 0 & 0 & 0 & $-\frac{c_{0}}{c_{1}}+\frac{c_{1} \xi^{2}}{4}$ \\
\hline$\frac{c_{1}^{2}}{4 c_{2}}$ & & $>0$ & 0 & 0 & $-\frac{c_{1}}{2 c_{2}}+\exp \left(\varepsilon \sqrt{c_{2}} \xi\right)$ \\
\hline 0 & & $<0$ & 0 & 0 & $-\frac{c_{1}}{2 c_{2}}+\frac{\varepsilon c_{1}}{2 c_{2}} \sin \left(\sqrt{-c_{2}} \xi\right)$ \\
\hline 0 & & $>0$ & 0 & 0 & $-\frac{c_{1}}{2 c_{2}}+\frac{\varepsilon \sqrt{ }-c_{1}^{2}}{2 c_{2}} \sinh \left(2 \sqrt{c_{2}} \xi\right)$ \\
\hline
\end{tabular}

Table 1. A series of fundamental solutions of (4).

Note: $g_{2}=-\frac{4 c_{1}}{c_{3}}$ and $g_{3}=-\frac{4 c_{0}}{c_{3}}$ are called invariants of the Weierstrass elliptic functions, $m$ is the modulus of the Jacobi elliptic functions; more detailed notations for the Weierstrass and Jacobi elliptic functions can be found in [5].

this case our proposed method becomes the extended tanh method [2]. If $c_{1}=c_{3}=0$, the results of (4) readily cover the results of the Jacobi function expansion method [3].

Remark 2. The proposed method not only gives a unified formulation to construct a series of exact solutions, but also provides a guideline to classify the types of solutions according to the given parameters. Furthermore, the proposed method is computerizable in solving nonlinear equations by using symbolic software like Maple or Mathematica.
In what follows we apply the generalized algebraic method to the $(1+1)$-dimensional variable coefficients MKdV equations and (2+1)-dimensional variable coefficients KP equations to illustrate our algorithm, which is more powerful than the tanh method and other sophisticated methods.

\section{3. (1+1)-Dimensional MKdV Equation with Variable Coefficients}

It is well known that the Korteweg-de Vries (KdV) equation plays an important role in soliton theory. 
Many properties of the KdV equation, such as symmetry, Bäcklund transformation, conservation laws, Lax pairs, Painleve analysis have been studied. Like the $\mathrm{KdV}$ equation, the variable coefficients $\mathrm{MKdV}$ equation [6]

$$
u_{t}+K_{1}\left(u_{x x x}+6 u^{2} u_{x}\right)+4 K_{2} u_{x}-K_{3}\left(u+x u_{x}\right)=0
$$

where $K_{1}=K_{1}(t), K_{2}=K_{2}(t), K_{3}=K_{3}(t)$ are arbitrary functions of only $t$, is also of importance in mathematical physics. The MKdV equation and the cylindrical MKdV equation etc. are special cases of $(5)[6,7]$.

Balancing the highest derivative term with the highest order nonlinear terms in (5) leads to $M=1$ or
$N=1$. Hence we may assume that

$$
\begin{aligned}
u(x, t)=A_{0}(x, t) & +A_{1}(x, t) P(\xi(x, t)) \\
& +B_{1}(x, t) / P(\xi(x, t)),
\end{aligned}
$$

where $A_{0}(x, t), A_{1}(x, t), \quad B_{1}(x, t), \quad P(\xi(x, t))$, and $\xi(x, t)=p(t) x+q(t)+\varphi$ are all differentiable functions, and $P(\xi)$ satisfies the ODE (4).

Substituting (6) along with (4) into (5), then setting the coefficients of each order of $P^{k}$ and $P^{k} \sqrt{\sum_{i=0}^{4} c_{i} P^{i}}$ (where $k=0,1, \ldots$ ) to zero, we get a set of overdetermined partial differential equations with respect to the unknown functions $A_{0}(x, t), A_{1}(x, t), B_{1}(x, t)$, $p(t)$, and $q(t)$. Solving the obtained system leads to the following three cases:

Case 1. $c_{1}=c_{3}=0$.

$$
\begin{aligned}
& A_{0}(x, t)=0, \quad A_{1}(x, t)=\alpha \exp \left(\int K_{3}(t) \mathrm{d} t\right), \quad B_{1}(x, t)=0, \quad p(t)=\mp \frac{\alpha \exp \left(\int K_{3}(t) \mathrm{d} t\right)}{\varepsilon \sqrt{-c_{4}}}, \\
& q(t)=\int \frac{\alpha \exp \left(\int K_{3}(t) \mathrm{d} t\right)\left( \pm \alpha^{2} K_{1}(t) c_{2} \exp \left(2 \int K_{3}(t) \mathrm{d} t\right) \mp 4 K_{2}(t) c_{4}\right)}{\varepsilon\left(-c_{4}\right)^{(3 / 2)}} \mathrm{d} t+\beta .
\end{aligned}
$$

Case 2. $c_{4}=0$.

$$
\begin{aligned}
& A_{0}(x, t)=\mp \frac{\alpha \varepsilon c_{1} \sqrt{-c_{0}} \exp \left(\int K_{3}(t) \mathrm{d} t\right)}{4 c_{0}}, \quad A_{1}(x, t)=0 \\
& B_{1}(x, t)=\mp \alpha \varepsilon \sqrt{-c_{0}} \exp \left(\int K_{3}(t) \mathrm{d} t\right), \quad p(t)=\alpha \exp \left(\int K_{3}(t) \mathrm{d} t\right) \\
& q(t)=\int \frac{\alpha\left(\alpha^{2} \varepsilon^{2}\left(-8 c_{0} c_{2} K_{1}(t)+3 c_{1}^{2} K_{1}(t)\right) \exp \left(2 \int K_{3}(t) \mathrm{d} t\right)-32 c_{0} K_{2}(t)\right)}{8 c_{0}} \exp \left(\int K_{3}(t) \mathrm{d} t\right) \mathrm{d} t+\beta
\end{aligned}
$$

Case 3. $c_{0}=c_{1}=0$.

$$
\begin{aligned}
& A_{0}(x, t)=\alpha \exp \left(\int K_{3}(t) \mathrm{d} t\right), \quad A_{1}(x, t)=\frac{4 c_{4} \alpha \exp \left(\int K_{3}(t) \mathrm{d} t\right)}{c_{3}} \\
& B_{1}(x, t)=0, \quad p(t)=\mp \frac{4 \alpha \sqrt{-c_{4}} \exp \left(\int K_{3}(t) \mathrm{d} t\right)}{\varepsilon c_{3}} \\
& q(t)=\mp \int \frac{8 \alpha \sqrt{-c_{4}}\left(\alpha^{2}\left(8 c_{4} c_{2} K_{1}(t)-3 c_{3}^{2} K_{1}(t)\right) \exp \left(2 \int K_{3}(t) \mathrm{d} t\right)-2 c_{3}^{2} K_{2}(t)\right)}{\varepsilon c_{3}^{3}} \exp \left(\int K_{3}(t) \mathrm{d} t\right) \mathrm{d} t+\beta
\end{aligned}
$$

and $\alpha$ and $\beta$ are integration constants.

According to Case 1, we get the following exact solutions of (5):

$$
\begin{aligned}
& u_{11}(x, t)=\varepsilon \sqrt{-\frac{c_{2}}{c_{4}}} A_{1}(x, t) \operatorname{sech}\left(\sqrt{c_{2}} \xi\right), \\
& c_{0}=0, \quad c_{2}>0, \quad c_{4}<0,
\end{aligned}
$$

$$
\begin{aligned}
u_{12}(x, t) & =\varepsilon \sqrt{-\frac{c_{2} m^{2}}{c_{4}\left(2 m^{2}-1\right)}} A_{1}(x, t) \mathrm{cn}\left(\sqrt{\frac{c_{2}}{2 m^{2}-1}} \xi\right), \\
c_{0} & =-\frac{c_{2}^{2} m^{2}\left(1-m^{2}\right)}{c_{4}\left(2 m^{2}-1\right)^{2}}, \quad c_{2}>0, \quad c_{4}<0, \quad(10 \mathrm{~b}) \\
u_{13}(x, t) & =\varepsilon \sqrt{-\frac{c_{2}}{c_{4}\left(2-m^{2}\right)}} A_{1}(x, t) \mathrm{dn}\left(\sqrt{\frac{c_{2}}{2-m^{2}}} \xi\right),
\end{aligned}
$$




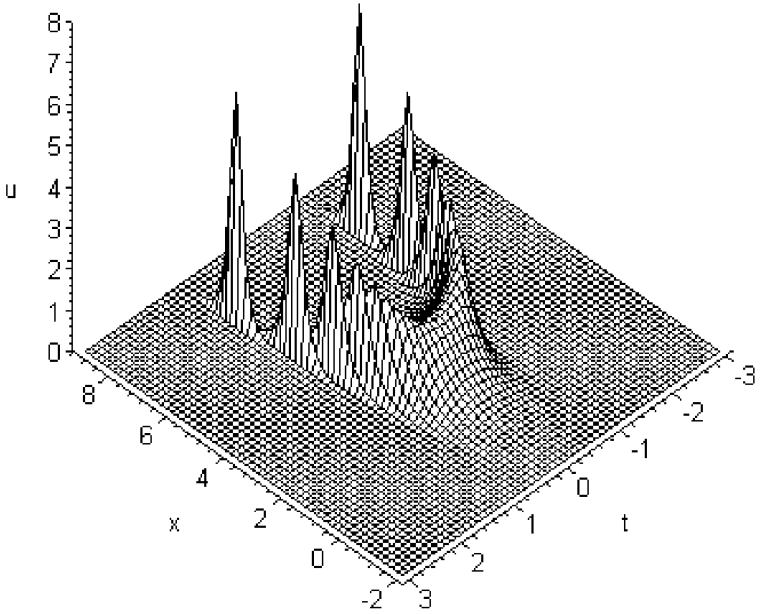

Fig. 1. The solution $u_{11}$ with parameters $c_{2}=0.5, c_{4}=$ -0.25 .

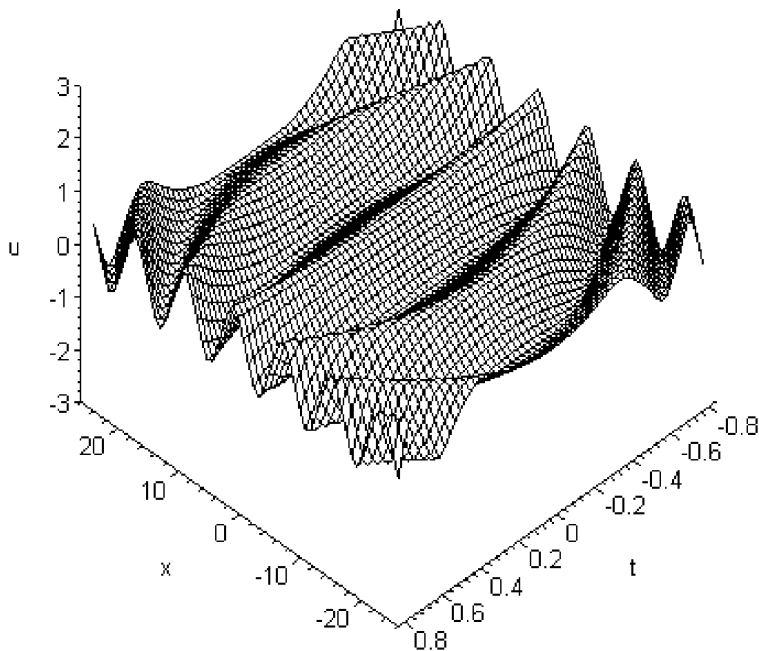

Fig. 2. The Jacobi doubly periodic solution $u_{12}$ with parameters $c_{2}=0.5, c_{4}=-16$.

$$
c_{0}=\frac{c_{2}^{2}\left(1-m^{2}\right)}{c_{4}\left(2-m^{2}\right)^{2}}, \quad c_{2}>0, \quad c_{4}<0
$$

where $\xi(x, t)=p(t) x+q(t)+\varphi$. The functions $A_{1}(x, t), p(t)$, and $q(t)$ are given in Case 1, (7). The plots for the solutions (10a), (10b) and (10c) are given in Figures 1, 2 and 3.

Case 2 gives the following solutions:

$$
\begin{aligned}
& u_{14}(x, t)=A_{0}(x, t)+\frac{B_{1}(x, t)}{\wp\left(\frac{\sqrt{c_{3}}}{2} \xi, g_{2}, g_{3}\right)}, \\
& c_{2}=0, \quad c_{3}>0, \quad g_{2}=-\frac{4 c_{1}}{c_{3}}, \quad g_{3}=-\frac{4 c_{0}}{c_{3}},
\end{aligned}
$$

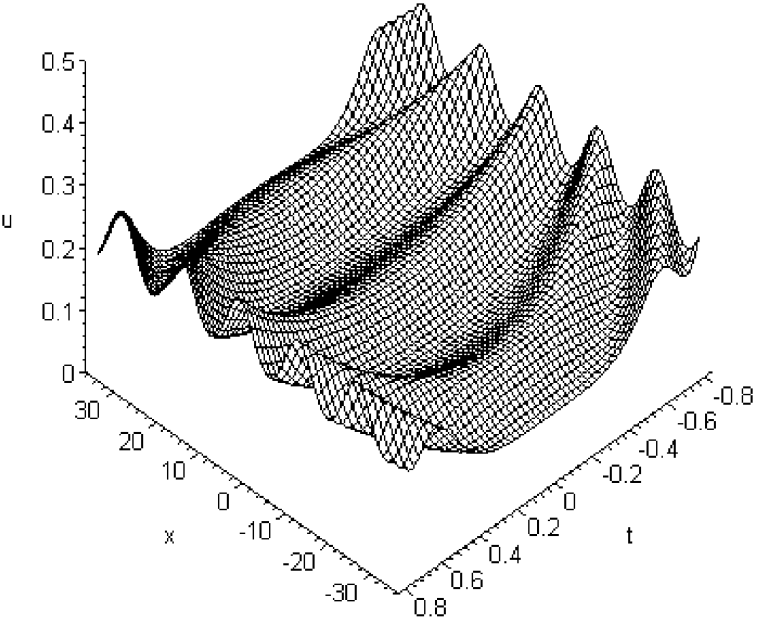

Fig. 3. The Jacobi doubly periodic solution $u_{13}$ with parameters $c_{2}=0.5, c_{4}=-16$.

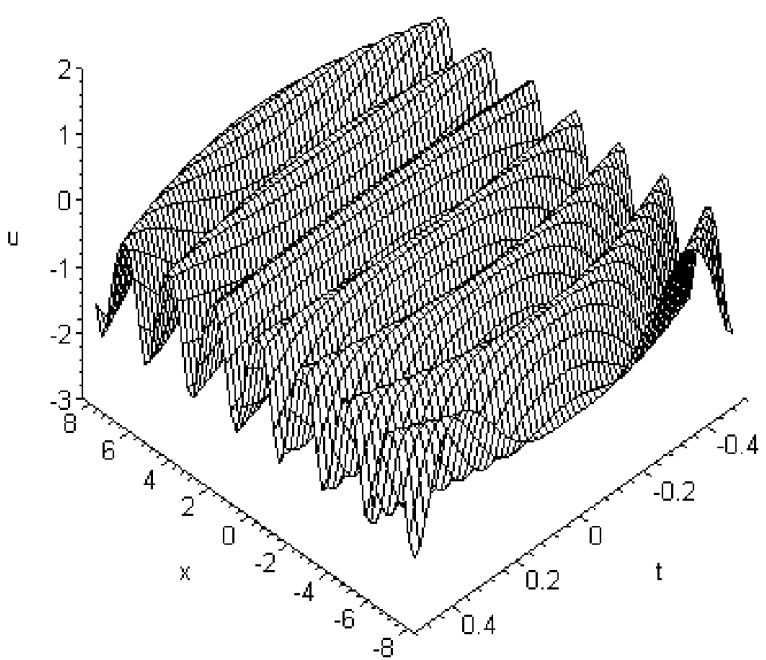

Fig. 4. The Weierstrass doubly periodic solution $u_{14}$ with parameters $c_{0}=c_{1}=-1$.

where $\xi(x, t)=p(t) x+q(t)+\varphi$. The functions $A_{0}(x, t), B_{1}(x, t), p(t)$, and $q(t)$ are given in Case 2, (8). The plot for the solutions (11) is given in Figure 4.

Finally, Case 3 leads to

$u_{15}(x, t)=$

$A_{0}(x, t)-A_{1}(x, t) \frac{c_{2} \sec ^{2}\left(\frac{1}{2} \sqrt{-c_{2}} \xi\right)}{2 \varepsilon \sqrt{-c_{2} c_{4}} \tan \left(\frac{1}{2} \sqrt{-c_{2}} \xi\right)+c_{3}}$,

$c_{2}<0$, 
$u_{16}(x, t)=A_{0}(x, t)$

$$
-A_{1}(x, t) \frac{c_{2} \operatorname{sech}^{2}\left(\frac{1}{2} \sqrt{c_{2}} \xi\right)}{2 \varepsilon \sqrt{c_{2} c_{4}} \tanh \left(\frac{1}{2} \sqrt{c_{2}} \xi\right)-c_{3}}, c_{2}>0,
$$

where $\xi(x, t)=p(t) x+q(t)+\varphi$. The functions $A_{0}(x, t), A_{1}(x, t), p(t)$, and $q(t)$ are given in Case 3, (9).

\section{4. (2+1)-Dimensional KP Equation with Variable Coefficients}

In this section we consider the (2+1)-dimensional variable coefficient generalized $\mathrm{KP}$ equation, which is a better integrable model,

$$
\begin{aligned}
& u_{x t}+h_{1}\left(u_{x x x}+6 u u_{x}+6 h_{3} u_{x}\right)_{x} \\
& \quad+h_{2} u_{y y}-\left(h_{3_{t}}+12 h_{1} h_{3}^{2}\right)=0,
\end{aligned}
$$

where $h_{1}=h_{1}(t), h_{2}=h_{2}(t)$ and $h_{3}=h_{3}(t)$ are all arbitrary functions with respect to $t$. When $h_{1}, h_{2}, h_{3}$ are given by special values, (13) reduces to many of the

Case 1: $\quad c_{1}=c_{3}=0, \quad c_{4} \neq 0$.

$$
\begin{aligned}
& u_{21}(x, y, t)=A_{0}(x, y, t)-\frac{c_{2}}{c_{4}} A_{2}(x, y, t) \operatorname{sech}^{2}\left(\sqrt{c_{2}} \xi\right), \quad c_{0}=0, \quad c_{2}>0, \quad c_{4}<0 \\
& u_{22}(x, y, t)=A_{0}(x, y, t)-\frac{c_{2}}{2 c_{4}} A_{2}(x, y, t) \tanh ^{2}\left(\sqrt{-\frac{c_{2}}{2}} \xi\right)-\frac{2 c_{4}}{c_{2}} \frac{B_{2}(x, y, t)}{\tanh ^{2}\left(\sqrt{-\frac{c_{2}}{2}} \xi\right)} \\
& u_{23}(x, y, t)=A_{0}(x, y, t)-\frac{c_{2}}{2 c_{4}} A_{2}(x, y, t) \tanh ^{2}\left(\sqrt{-\frac{c_{2}}{2}} \xi\right) \\
& u_{24}(x, y, t)=A_{0}(x, y, t)-\frac{2 c_{4}}{c_{2}} \frac{B_{2}(x, y, t)}{\tanh ^{2}\left(\sqrt{-\frac{c_{2}}{2}} \xi\right)}
\end{aligned}
$$

famous nonlinear wave equations, such as the $\mathrm{KdV}$ equation $\left[h_{1}=1, h_{2}=h_{3}=0\right.$, and integrating (13) with respect to $x]$, the generalized $\mathrm{KdV}$ equation $\left[h_{1}=1\right.$, $h_{2}=0$, and integrating (13) with respect to $x$ ], the KP equation $\left(h_{1}=h_{2}=1, h_{3}=0\right)$ and the generalized KP equation $\left(h_{1}=1\right)$ [7-9].

Balancing the highest derivative term with the highest order nonlinear term in (13) leads to $M=2$ or $N=2$. We thus may assume that

$$
\begin{aligned}
u(x, y, t)= & A_{0}(x, y, t)+A_{1}(x, y, t) P(\xi(x, y, t)) \\
& +B_{1}(x, y, t) / P(\xi(x, y, t)) \\
& +A_{2}(x, y, t) P^{2}(\xi(x, y, t)) \\
& +B_{2}(x, y, t) / P^{2}(\xi(x, y, t)),
\end{aligned}
$$

where $A_{0}(x, y, t), \quad A_{1}(x, y, t), \quad B_{1}(x, y, t), \quad A_{2}(x, y, t)$, $B_{2}(x, y, t), \quad P(\xi(x, y, t))$, and $\xi(x, y, t)=p(y, t) x+$ $q(y, t)+\varphi$ are all differentiable functions and $P(\xi)$ satisfies the ODE (4).

Similar to the solving process of the above $(1+1)$ dimensional variable coefficients $\mathrm{MKdV}$ equation, we obtain the following exact solutions of (13):

where $A_{2}(x, y, t)=-2 c_{4}\left(\exp \left(\int F(t) \mathrm{d} t+\alpha\right)\right)^{2}, B_{2}(x, y, t)=-2 c_{0}\left(\exp \left(\int F(t) \mathrm{d} t+\alpha\right)\right)^{2}, c_{2}<0, c_{4}>0, c_{0}=$ $\frac{c_{2}^{2}}{4 c_{4}}, \xi(x, y, t)=p(y, t) x+q(y, t)+\varphi$. Here we omit the concrete expressions of $A_{0}(x, y, t), p(y, t)$, and $q(y, t)$ for complexity.

$$
\begin{gathered}
u_{25}(x, y, t)=A_{0}(x, y, t)+A_{2}(x, y, t) \frac{1}{c_{4} \xi^{2}}, \quad c_{0}=0, \quad c_{2}=0, \quad c_{4}>0, \\
u_{26}(x, y, t)=A_{0}(x, y, t)-\frac{c_{2} m^{2}}{c_{4}\left(2 m^{2}-1\right)} A_{2}(x, y, t) \mathrm{cn}^{2}\left(\sqrt{\frac{c_{2}}{2 m^{2}-1}} \xi\right)-\frac{c_{4}\left(2 m^{2}-1\right)}{c_{2} m^{2}} \frac{B_{2}(x, y, t)}{\mathrm{cn}^{2}\left(\sqrt{\frac{c_{2}}{2 m^{2}-1}} \xi\right)}, \\
u_{27}(x, y, t)=A_{0}(x, y, t)-\frac{c_{2} m^{2}}{c_{4}\left(2 m^{2}-1\right)} A_{2}(x, y, t) \mathrm{cn}^{2}\left(\sqrt{\frac{c_{2}}{2 m^{2}-1}} \xi\right),
\end{gathered}
$$


(a)

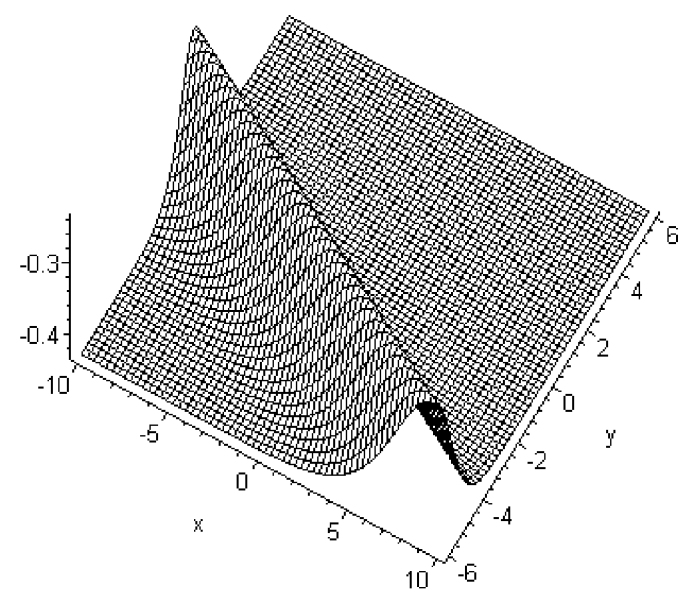

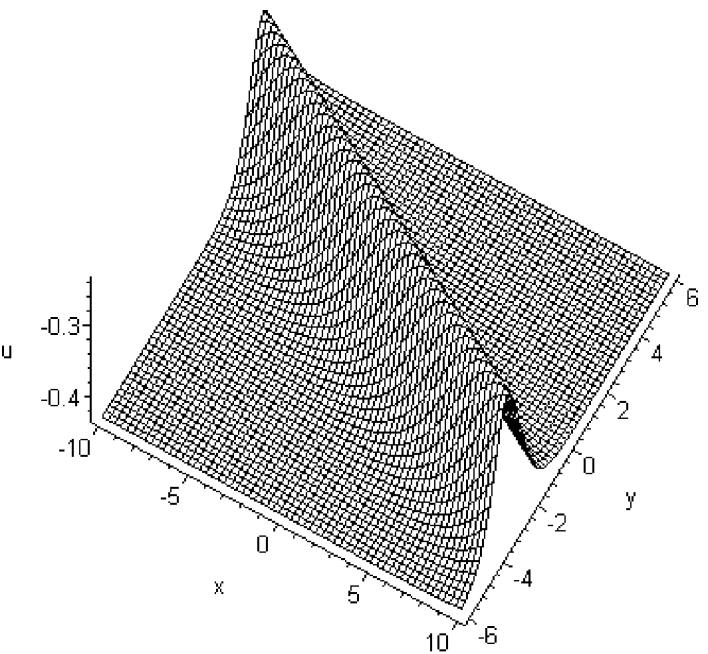

(b)

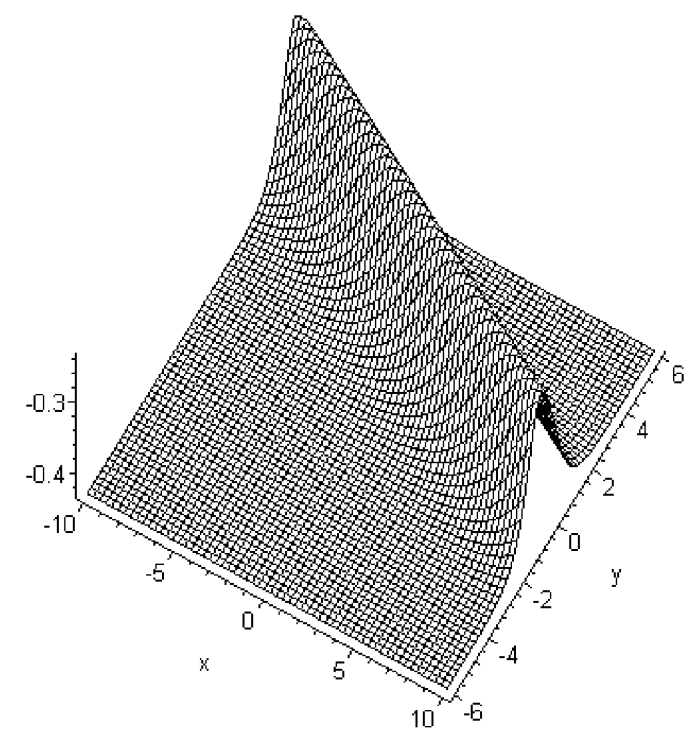

Fig. 5. The asymptotical property of the soliton solution $u_{21}$.

$$
u_{28}(x, y, t)=A_{0}(x, y, t)-\frac{c_{4}\left(2 m^{2}-1\right)}{c_{2} m^{2}} \frac{B_{2}(x, y, t)}{\mathrm{cn}^{2}\left(\sqrt{\frac{c_{2}}{2 m^{2}-1}} \xi\right)}
$$

where $A_{2}(x, y, t)=-2 c_{4}\left(\exp \left(\int F(t) \mathrm{d} t+\alpha\right)\right)^{2}, B_{2}(x, y, t)=-2 c_{0}\left(\exp \left(\int F(t) \mathrm{d} t+\alpha\right)\right)^{2}, c_{2}>0, c_{4}<0, c_{0}=$ $-\frac{c_{2}^{2} m^{2}\left(1-m^{2}\right)}{c_{4}\left(2 m^{2}-1\right)^{2}}, \xi(x, y, t)=p(y, t) x+q(y, t)+\varphi$.

Remark 3. Here we have omitted the solutions in terms of $\sec \xi, \tan \xi, \operatorname{dn} \xi$ and $\operatorname{sn} \xi$. The Jacobi periodic solution degenerates to the soliton solution when $m \rightarrow 1$. It is well known that soliton solutions and Ja- cobi doubly periodic solutions are interesting and of physical relevance. Here we take the solutions (15a) and $(15 \mathrm{~g})$ as samples to further analyze their properties by some figures (Figs. 5 and 6). When $t$ goes from 
(a)

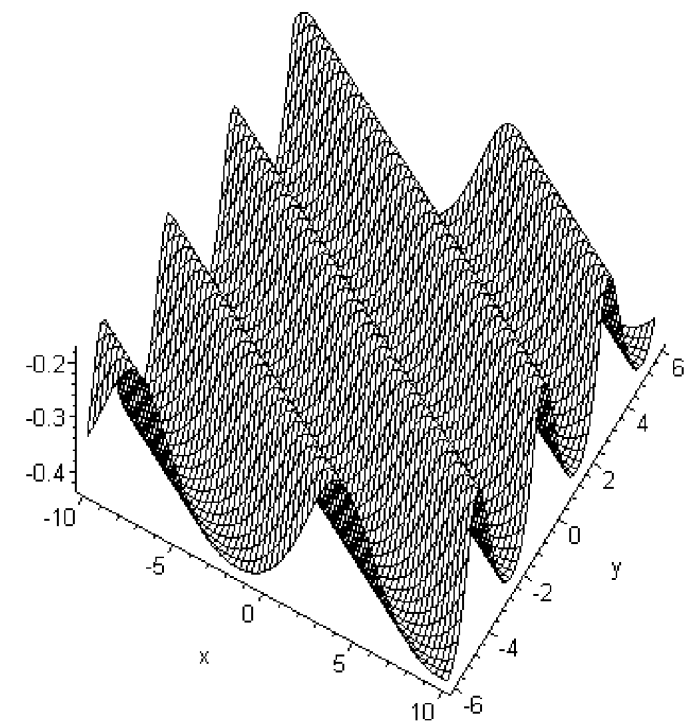

(b)

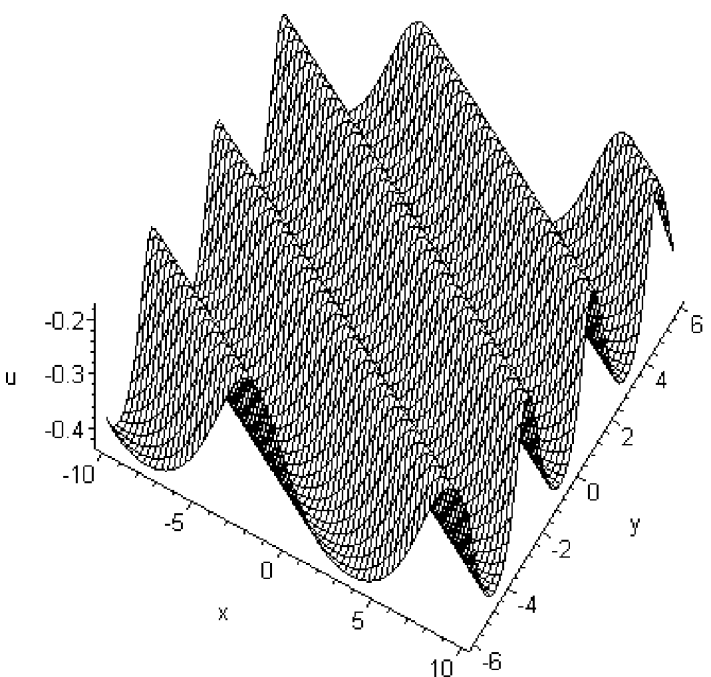

(c)

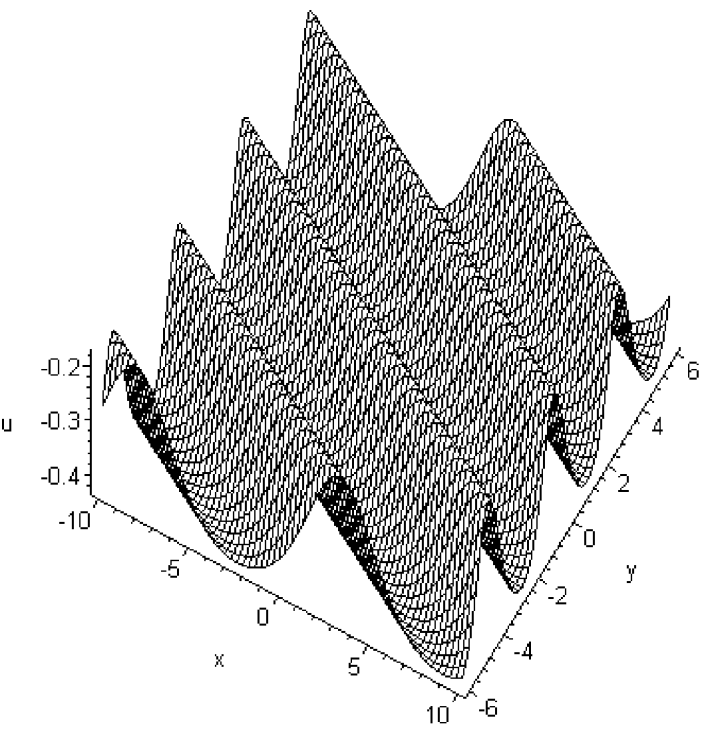

Fig. 6. The asymptotical property of the Jacobi doubly periodic wave solution $u_{27}$. $-\infty$ to $+\infty$, they form their limit positions at $t=-\infty$ asymptotically tends to their limit positions at $t=+\infty$.
The properties are similar to the asymptotically standing soliton.

Case 2. $\quad c_{4}=0$.

$$
\begin{aligned}
& u_{29}(x, y, t)=A_{0}(x, y, t)-\frac{c_{2}}{c_{3}} A_{1}(x, y, t) \operatorname{sech}^{2}\left(\frac{\sqrt{c_{2}}}{2} \xi\right), \quad c_{0}=c_{1}=0, \quad c_{2}>0, \\
& u_{210}(x, y, t)=A_{0}(x, y, t)-\frac{c_{2}}{c_{3}} A_{1}(x, y, t) \sec ^{2}\left(\frac{\sqrt{-c_{2}}}{2} \xi\right), \quad c_{0}=c_{1}=0, \quad c_{2}<0, \\
& u_{211}(x, y, t)=A_{0}(x, y, t)+A_{1}(x, y, t) \frac{1}{c_{3} \xi^{2}}, \quad c_{0}=c_{1}=c_{2}=0,
\end{aligned}
$$


(a)
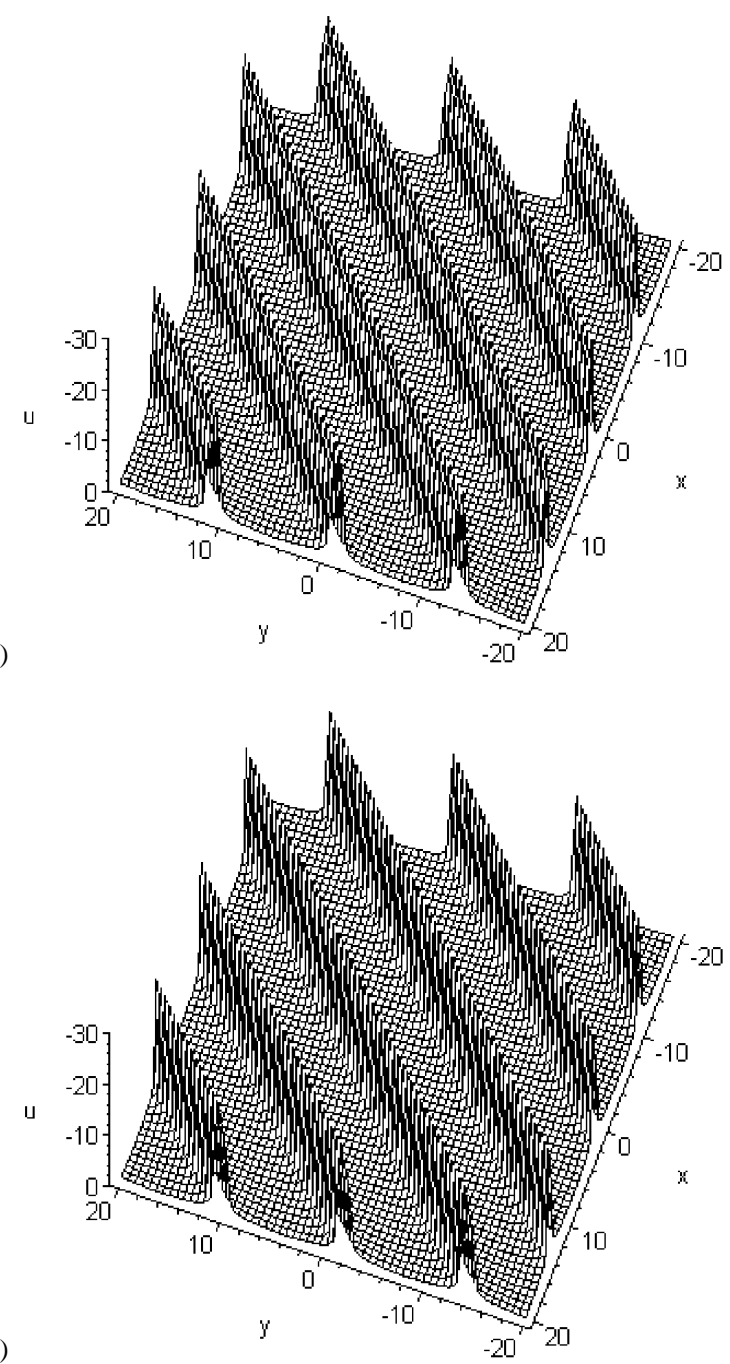

(b)

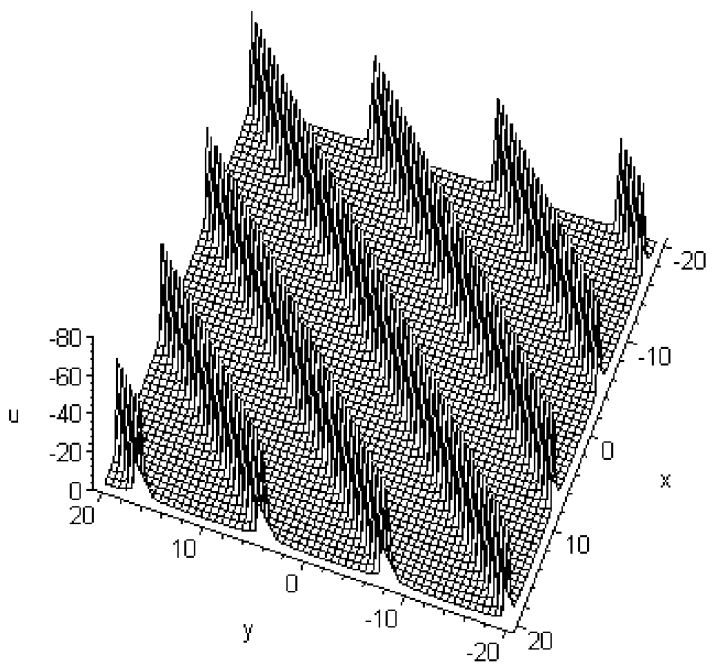

Fig. 7. The asymptotical property of the Weierstrass doubly periodic wave solution $u_{212}$.

$$
u_{212}(x, y, t)=A_{0}(x, y, t)+A_{1}(x, y, t) \wp\left(\frac{\sqrt{c_{3}}}{2} \xi, g_{2}, g_{3}\right) \text {, }
$$

where $c_{2}=0, c_{3}>0, g_{2}=-\frac{4 c_{1}}{c_{3}}, g_{3}=-\frac{4 c_{0}}{c_{3}}, \xi(x, y, t)=p(y, t) x+q(y, t)+\varphi$. The asymptotical behavior of the Weierstrass doubly periodic wave solution (16d) is shown in Figure 7.

\section{Conclusion}

In summary, a new generalized algebraic method with computerized symbolic computation is developed to deal with variable coefficients' NEEs. Then, when applying the proposed method to (5) and (13), a rich variety of exact explicit solutions is obtained. As indicated before, in Remark 1, the proposed algorithm is more general than the tanh method [1], the ex- tended tanh method [2], the Jacobi function expansion method [3], and the algebraic method [4]. Moreover, our method can also be applied to constant coefficients NEEs.

\section{Acknowledgement}

The authors would like to express their sincere thanks to the referees for their many helpful advices 
and suggestions. This work was supported by the National Natural Science Foundation of China and the

[1] a) L. Huibin and W. Kelin, J. Phys. A: Math. Gen. 23, 4097 (1990); b) W. Malfliet, Am. J. Phys. 60, 650 (1992).

[2] a) Y. T. Gao and B. Tian, Comput. Math. Appl. 33, 115 (1997); b) S. A. Elwakil, S. K. El-labany, and M. A. Zahran, Phys. Lett. A 299, 179 (2002); c) W. X. Ma, Int. J. Nonlinear Mech. 31, 329 (1996); d) E. G. Fan, Phys. Lett. A 277, 212 (2000); e) Z. Y. Yan and H. Q. Zhang, Phys. Lett. A 285, 355 (2001); f) C.L. Bai and H. Zhao, Commun. Theor. Phys. 41, 251 (2004); g) C. L. Bai and H. Zhao, Commun. Theor. Phys. 42, 189 (2004); h) H. Zhao and C. L. Bai, Commun. Theor. Phys. 43, 251 (2005).

[3] a) S. K. Liu, Z.T. Fu, S. D. Liu, and Q. Zhao, Phys.
Natural Science Foundation of Shandong Province in China.

Lett. A 289, 69 (2001); b) Z. T. Fu, S. K. Liu, and S. D. Liu, Phys. Lett. A 290, 72 (2001).

[4] E. G. Fan, Chaos, Solitons and Fractals 16, 819 (2003).

[5] N.L. Akhiezer, Elements of Theory of Elliptic Functions, American Mathematical Society, Providence, RI 1990.

[6] S. Y. Lou and H.R. Ruan, Acta Phys. Sin. 41, 182 (1992) (in Chinese).

[7] M. J. Ablowitz and P. A. Clarkson, Solitons, Nonlinear Evolution Equations and Inverse Scattering, Cambridge University Press, Cambridge 1991.

[8] T. Brugarino, J. Math. Phys. 30, 1013 (1989).

[9] M. R. Miura, Bäcklund Transformation, SpringerVerlag, Berlin 1978. 